\title{
SUPPLEMENTARY INFORMATION: Label-Free Optical Method for Quantifying Molecular Transport Across Cellular Membranes In Vitro
}

Mohammad Sharifian Gh., Michael J. Wilhelm*, and Hai-Lung Dai

Department of Chemistry, Temple University, 1901 N. $13^{\text {th }}$ Street, Philadelphia, PA 19122, USA.

* Author to whom correspondence should be addressed. E-mail: michael.wilhelm@temple.edu

CONTENTS:

1. METHODS

2. CONTROL EXPERIMENTS: CHECK FOR PHOTOINDUCED DAMAGE

3. LANGMUIR ADSORPTION ISOTHERM ASSAY

4. ONE-SITE CHANNEL MODEL OF COMPETITIVE TRANSPORT

\section{METHODS}

The Gram-negative bacteria, Escherichia (E.) coli (mc4100 strain, ATCC 35695), was cultivated on Lauria Broth agar (Sigma-Aldrich) medium plates at $37^{\circ} \mathrm{C}$ for ca. 24 hours and then stored at $4{ }^{\circ} \mathrm{C}$ for future use. Discrete colonies of $E$. coli were grown aerobically at $37^{\circ} \mathrm{C}$ in $50 \mathrm{~mL}$ Terrific Broth (TB, Cat. No. T0918, Sigma-Aldrich) solution in a shaking flask at $150 \mathrm{rpm}$ for ca. 16-18 hours - to late exponential phase. The harvested cells were centrifuged (1500xg, $10 \mathrm{~min}$, room temperature) and then washed with sufficient 1XPBS two times to remove waste and residual TB. For each washing step, we used a Rotamix (10101-RKVSD, ATR Inc.) rotator (ca. 35-40 rpm) to re-suspend the pellet of cells in 1xPBS. After washing, the pellets were combined and suspended in $1 \mathrm{xPBS}$ to a final cell density of ca. $(6.0 \pm 0.1) \times 10^{7}$ cells. $\mathrm{mL}^{-1}$.

Stock solutions of malachite green oxalate (Sigma Aldrich) and propidium iodide, PI (Cat. No.: P4170, Sigma-Aldrich), with concentration of $500 \mu \mathrm{M}$, were prepared in distilled deionized water (Millipore, 18.2 M. $\mathrm{cm}$ ) and stored in the dark at $4^{0} \mathrm{C}$ for future use. For each SHS experiment, an appropriate amount of each stock was used to achieve the final concentration of $5 \mu \mathrm{M} \mathrm{MG}$, and either $5,50,100,150$, or $200 \mu \mathrm{M}$ Pro in the liquid flow jet.

Our SHS experimental setup has been described previously in detail ${ }^{1}$. The setup consists of a mode-locked Ti:Sapphire laser (Coherent, Micra V, oscillator only) tuned to $(800 \pm 10) \mathrm{nm}$ and delivering pulses of ca. $50 \mathrm{fs}$ at a repetition rate of $76 \mathrm{MHz}$ with $\sim 4$ $\mathrm{nJ}$ pulse energy. The average output power of the laser is ca. 280-300 mW. A long-pass filter (schott, RG695) was placed before the objective lens to remove any residual light generated at the second harmonic frequency prior to the sample. The sample flow 
system used for SHS measurements was reported previously ${ }^{1}$. We added a small amount of cell stock suspension $(0.50 \mathrm{~mL}$ ) into the flowing Pro or MG + Pro solutions (at $\mathrm{t}=0 \mathrm{~s}$ ) to achieve the appropriate final concentration of $5-200 \mu \mathrm{M}$ of Pro, $5 \mu \mathrm{M}$ of MG, and $(3.0 \pm 0.1) \times 10^{6}$ cells. $\mathrm{mL}^{-1}$ of $E$. coli. All solutions were allowed to equilibrate to room temperature (ca. 293K) prior to the experiment. The second harmonic light (ca. $400 \mathrm{~nm}$ ) was collected in the forward scattering direction after passing through a collective lens, a BG39 band-pass filter, and finally a monochromator. A photomultiplier tube (Hamamatsu, R585) was used to detect the $\mathrm{SH}$ photons. The $\mathrm{SH}$ signal passed through a pre-amplifier (Stanford Research System, SR440), and was processed through a correlated photon counting system (Stanford Research System, SRS SR400). Signal was collected in 1 second intervals, with a gate time of 0.5 seconds. The $\mathrm{SH}$ signal was recorded at $(400 \pm 5) \mathrm{nm}$.

\section{CONTROL EXPERIMENTS: CHECK FOR PHOTOINDUCED DAMAGE}

For molecules which absorb in the visible (i.e., dyes), time-resolved optical bright-field transmission microscopy (TM) permits a complementary means to measure molecular uptake kinetics ${ }^{1}$ without application of a scattering laser; and can therefore be used as a controlled test for photo-induced damage to the cell membrane. Specifically, if the scattering laser used in the SHS experiments induces damage to the cell membrane(s), the measured transport rate(s) will be artificially increased relative to the rate(s) measured by time-resolved TM. Figure $\mathrm{S} 1$ depicts a direct comparison of the deduced uptake kinetics for Pro by living E. coli measured using both time-resolved TM and SHS.

Figure S1. Comparison of the measured Pro uptake kinetics into living $E$. coli deduced with time-resolved TM (blue open circles) and SHS (grey filled circles).

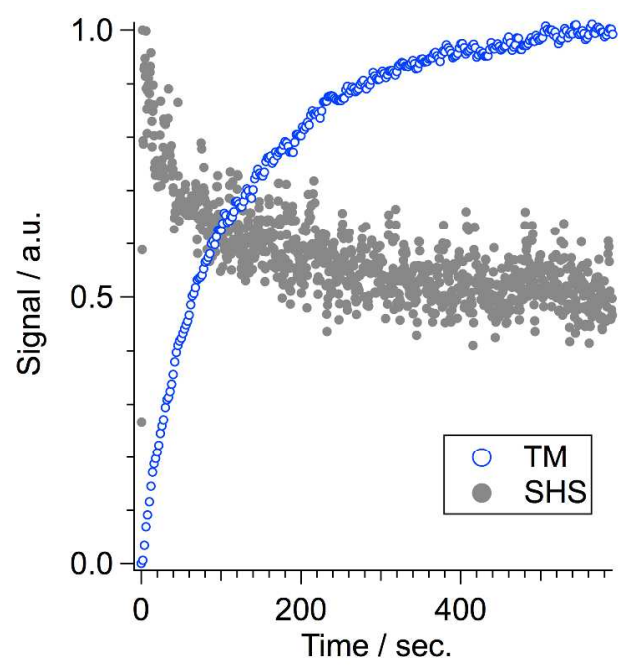


Note, in live cells, Pro is unable to cross the cytoplasmic membrane (CM), and (for Gram-negative bacteria) is therefore restricted to sampling the periplasmic space bound by the cell's outer membrane (OM) and the CM. Correspondingly, the SHS signal shows a rise and decay indicative of adsorption onto the outer surface of the OM (rise), transport across the Omp, and adsorption onto the inner surface of the OM (decay). Conversely, the TM signal, deduced as the resulting ensemble average signal (i.e., $n=20$ live cells), which were individually measured from the set of TM images as the total absorption signal from circular regions-of-interest (ROI) centered in representative live cells, shows a monotone rise of signal as Pro diffuses into and concentrates within the periplasmic space of the bacteria cell (i.e., Beer's law). Subsequently, the rate of the signal rise in the TM experiment, and the rate of the signal decay in the SHS experiment are complementary measures of the rate of saturation $\left(\mathrm{k}_{\mathrm{sat}}\right)$ of Pro within the periplasmic space of $E$. coli. Analysis of the measured signals reveals deduced values of $k_{\text {sat }}$ corresponding to: $0.009 \pm 0.0002 \mathrm{~s}^{-1}$ (TM) and $0.01 \pm 0.001 \mathrm{~s}^{-1}$ (SHS), which are effectively identical (within experimental error). It is therefore reasonable to conclude that there is little to no laser-induced damage to the bacterial cell membranes in the SHS experiments.

\section{LANGMUIR ADSORPTION ISOTHERM ASSAY}

$\mathrm{SH}$ signal resulting from adsorption of a molecule-of-interest onto a surface is proportional to the square of the coverage of the molecule on the surface which, in the case of MG or Pro adsorbed onto the E. coli OM, can be generally represented as follow ${ }^{2,3}$;

$$
\mathrm{I}_{\mathrm{SH}}=\gamma+\left(\beta_{\text {cell }}^{(2)}+\beta_{\text {mol. }}^{(2)} \theta_{\text {cell }}^{\text {mol. }} \times \mathrm{e}^{i \varphi}\right)^{2},
$$

where $\gamma, \beta_{c e l l}^{(2)}$, and $\beta_{m o l}^{(2)}$ represent, respectively, all background contributions, the nonlinear optical polarization associated with uncoated E. coli, and the unit nonlinear optical polarization from the surface adsorbed molecule-of-interest; $\theta_{\text {cell }}^{\text {mol. }}$ represents the molecular surface coverage; and $\mathrm{e}^{i \varphi}$ takes into account the relative phase difference (i.e. $\varphi)$ between the interfering nonlinear polarizations from the bare cell surface and the molecule on the surface. Control experiments run without the addition of either MG or Pro reveal that the nonlinear polarization of bare bacterial cells is effectively zero. Subsequently, in experiments with cells and either MG or Pro, the surface bound 
molecules are the sole source of nonlinear polarization, hence equation (S1) can be simplified as:

$$
\mathrm{I}_{\mathrm{SH}} \cong \gamma+\left(\beta_{\text {mol. }}^{(2)} \theta_{\text {cell }}^{\text {mol. }}\right)^{2}
$$

where the phase difference term can be dropped as there is effectively only a single source of nonlinear polarization. By assuming that the surface adsorption of the molecules follows Langmuir kinetics, we then can apply the $\mathrm{pH}$-adjusted modified Langmuir isotherm model as follows ${ }^{3}$;

$$
\theta_{\text {cell }}^{\text {mol. }}=\frac{C_{\text {mol. }}+N_{\text {max }}^{\text {mol }}+\frac{55.5}{\alpha K_{D}}-\sqrt{\left(C_{\text {mol. }}+N_{\text {max }}^{\text {mol. }}+\frac{55.5}{\alpha K_{D}}\right)^{2}-4 C_{\text {mol. }} N_{\text {max }}^{\text {mol. }}}}{2 N_{\text {max }}^{\text {mol. }}},
$$

where

$$
\alpha=\frac{\left[\mathrm{H}^{+}\right]}{\left[\mathrm{H}^{+}\right]+K_{a}^{\text {mol. }}},
$$

where $C_{m o l}, N_{m a x}^{m o l}, K_{a}^{m o l}$, and $K_{D}$ represent, respectively, the total concentration of the molecule of interest, the maximum number density of the surface adsorbed molecules, the acidic ionization constant of the molecule, and the adsorption equilibrium constant of the molecule. The Langmuir isotherm was constructed by plotting the maximum intensity of the initial transport peak as a function of concentration (Figure $1 \mathrm{~b}$, right). The isotherm can be analyzed by fitting to equation (S2), which yields the non-linear optical polarizability of the molecule (i.e. $\beta_{m o l}^{(2)}$ ). Analysis of the adsorption isotherms for MG and Pro yield the following relationship:

$$
\beta_{P r o}^{(2)} \cong 0.04 \times \beta_{M G}^{(2)}
$$

It should be noted that molecular surface orientation effects have not been taken into account in this analysis. The nonlinear polarizability defined by equation S5 is therefore valid solely for comparing MG and Pro adsorbed on the OM surface of $E$. coli.

\section{ONE-SITE CHANNEL MODEL OF COMPETITIVE TRANSPORT}

We employ a modification to the previously established one-site channel competitive transport model of Zilman et al. ${ }^{4}$ Briefly, by combining the time-dependent probability 
states of the channel (i.e., the channel is either occupied with the reference species "reference", the target species "target", or it is empty), the perturbed transport rate of the reference species crossing from the exoplasmic to the periplasmic space in the copresence of the target species, $k_{\rightarrow}^{\text {Ref }\{\text { Target }\}}$, can be expressed as:

$$
k_{\rightarrow}^{\text {Ref }\{\text { Target }\}}=\frac{\mathrm{r}_{\rightarrow}^{\text {Ref }} \mathrm{R}_{\rightarrow}^{\text {Ref }}}{\mathrm{r}_{\rightarrow}^{\text {Ref }}+\mathrm{r}_{\leftarrow}^{\text {Ref }}+\mathrm{R}_{\rightarrow}^{\text {Ref }}+\left(\frac{\mathrm{r}_{\rightarrow}^{\text {Ref }}+\mathrm{r}_{\leftarrow}^{\text {Ref }}}{\mathrm{r}_{\rightarrow}^{\text {Target }}+\mathrm{r}_{\leftarrow}^{\text {Target }}}\right) \mathrm{R}_{\rightarrow}^{\text {Target }}},
$$

where $r_{\rightarrow}$ and $r_{\leftarrow}$ are the forward and reverse rates for exiting the channel, and $R_{\rightarrow}$ is the forward rate for entering the channel (Figure 3a). Similarly, the unperturbed transport rates for the reference and target species can be expressed as:

$$
k_{\rightarrow}^{R e f}=\frac{\mathrm{r}_{\rightarrow}^{\text {Ref }} \mathrm{R}_{\rightarrow}^{\text {Ref }}}{\mathrm{r}_{\rightarrow}^{\text {Ref }}+\mathrm{r}_{\leftarrow}^{\text {Ref }}+\mathrm{R}_{\rightarrow}^{\text {Ref }}}
$$

and

$$
k_{\rightarrow}^{\text {Target }}=\frac{\mathrm{r}_{\rightarrow}^{\text {Target }} \mathrm{R}_{\rightarrow}^{\text {Target }}}{\mathrm{r}_{\rightarrow}^{\text {Target }}+\mathrm{r}_{\leftarrow}^{\text {Target }}+\mathrm{R}_{\rightarrow}^{\text {Target }}} .
$$

Under the initial non-equilibrium conditions of our experiment, the concentrations of the reference and target molecules in the exoplasmic space are much greater than in the periplasmic space, subsequently it is reasonable to assume that the forward exit rate out of the channel is much faster than the reverse exit rate, i.e., $r_{\rightarrow}^{x} \gg r_{\leftarrow}^{x}$. Equations S6-S8 can therefore be simplified as:

$$
\begin{gathered}
k_{\rightarrow}^{\text {Ref }\{\text { Target }\}} \cong \frac{\mathrm{r}_{\rightarrow}^{\text {Ref }} \mathrm{R}_{\rightarrow}^{\text {Ref }}}{\mathrm{r}_{\rightarrow}^{\text {Ref }}+\mathrm{R}_{\rightarrow}^{\text {Ref }}+\left(\frac{\mathrm{r}_{\rightarrow}^{\text {Ref }}}{\mathrm{r}_{\rightarrow}^{\text {Target }}}\right) \mathrm{R}_{\rightarrow}^{\text {Target }}}, \\
k_{\rightarrow}^{\text {Ref }} \cong \frac{\mathrm{r}_{\rightarrow}^{\text {Ref }} \mathrm{R}_{\rightarrow}^{\text {Ref }}}{\mathrm{r}_{\rightarrow}^{\text {Ref }}+\mathrm{R}_{\rightarrow}^{\text {Ref }}},
\end{gathered}
$$




$$
k_{\rightarrow}^{\text {Target }} \cong \frac{\mathrm{r}_{\rightarrow}^{\text {Target }} \mathrm{R}_{\rightarrow}^{\text {Target }}}{\mathrm{r}_{\rightarrow}^{\text {Target }}+\mathrm{R}_{\rightarrow}^{\text {Target }}}
$$

Ultimately, it is of interest to develop an expression for the forward transport rate of the target species, $k_{\rightarrow}^{\text {Target }}$, with respect to relevant experimental parameters. By combining equations S9-S11, and following some simple rearrangement, we obtain:

$$
k_{\rightarrow}^{\text {Target }} \cong \frac{k_{\rightarrow}^{\text {Ref }} k_{\rightarrow}^{\text {Ref }\{\text { Target }\}} \mathrm{R}_{\rightarrow}^{\text {Ref }}}{\left(k_{\rightarrow}^{\text {Ref }} k_{\rightarrow}^{\text {Ref }\{\text { Target }\}}\right)+\left(k_{\rightarrow}^{\text {Ref }}-k_{\rightarrow}^{\text {Ref }\{\text { Target }\}}\right) \mathrm{R}_{\rightarrow}^{\text {Ref }}}\left(\frac{\mathrm{R}_{\rightarrow}^{\text {Target }}}{\mathrm{R}_{\rightarrow}^{\text {Ref }}}\right)
$$

Further, for large concentration ratios (i.e., [target]/[reference]), it is reasonable to assume that $\mathrm{R}_{\rightarrow} \gg \mathrm{R}_{\leftarrow}$ (i.e., due to the diffusion gradient) and that $k_{\rightarrow}^{\text {Ref }\{\text { Target }\}}$ approaches zero (i.e., maximum possible perturbation). Subsequently, equation S12 can be further simplified to:

$$
k_{\rightarrow}^{\text {Target }} \cong \frac{k_{\rightarrow}^{\text {Ref }} k_{\rightarrow}^{\text {Ref }\{\text { Target }\}}}{\left(k_{\rightarrow}^{\text {Ref }}-k_{\rightarrow}^{\text {Ref }\{\text { Target }\}}\right)}\left(\frac{\mathrm{R}_{\rightarrow}^{\text {Target }}}{\mathrm{R}_{\rightarrow}^{\text {Ref }}}\right)
$$

Both $\mathrm{k}_{\rightarrow}^{R e f}$ and $k_{\rightarrow}^{R e f\{\text { Target }\}}$ are directly measurable quantities. Further, the ratio of rates in equation (S13), can be directly related to experimental parameters:

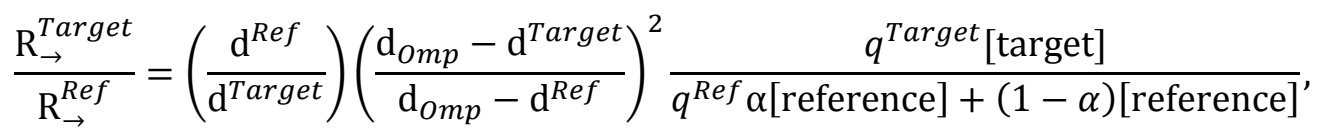

where $\mathrm{d}^{\text {Ref }}, \mathrm{d}^{\text {Target }}$, and $\mathrm{d}_{\text {omp }}$ are the short-axis diameters of the reference molecule, the target molecule, and the channel lumen, respectively; $q^{\text {Ref }}$ and $q^{\text {Target }}$ are the charges of the reference and target molecules; and [reference] and [target] are the concentrations of the reference and target molecules, respectively. In general, the first term on the right hand side of equation (S14) is the ratio of the corresponding diffusion constants, the second term compares the cross-sectional area of the channel and the diffusing molecule, and the third term accounts for Coulombic repulsion between the 
reference and target species. Note that when $M G$ is used as the reference molecule, given that $\mathrm{MG}$ is $\mathrm{pH}$ sensitive, only a fraction: i.e.,

$$
\alpha=\frac{\left[\mathrm{H}^{+}\right]}{\left[\mathrm{H}^{+}\right]+K_{a}}
$$

of $M G$ exists in cationic form - and so only $\alpha[M G]$ contributes to the repulsive interaction. Conversely, under the applied experimental conditions, Pro exists purely as a $2+$ cationic species. However, if the target molecule exhibited $\mathrm{pH}$ sensitivity, a similar correction factor would need to be applied. Nevertheless, regardless of the charge state, if both neutral and cationic species can cross the channel, both would contribute to the overall competitive transport rate. Subsequently, the total concentration must be taken into account in the rate expression.

\section{REFERENCES}

(1) Wilhelm, M. J.; Sheffield, J. B.; Gonella, G.; Wu, Y.; Spahr, C.; Zeng, J.; Xu, B.; Dai, H.-L. Real-Time Molecular Uptake and Membrane-Specific Transport in Living Cells by Optical Microscopy and Nonlinear Light Scattering. Chem. Phys. Lett. 2014, 605-606, 158-163.

(2) Wang, H.-F.; Troxler, T.; Yeh, A.-G.; Dai, H.-L. In Situ, Nonlinear Optical Probe of Surfactant Adsorption on the Surface of Microparticles in Colloids. Langmuir 2000, 16, 2475-2481.

(3) Zeng, J.; Eckenrode, H. M.; Dai, H.-L.; Wilhelm, M. J. Adsorption and Transport of Charged vs. Neutral Hydrophobic Molecules at the Membrane of Murine Erythroleukemia (MEL) Cells. Colloids Surf. B Biointerfaces 2015, 127, 122-129.

(4) Zilman, A.; di Talia, S.; Jovanovic-Talisman, T.; Chait, B. T.; Rout, M. P.; Magnasco, M. O. Enhancement of Transport Selectivity Through Nano-Channels by Non-Specific Competition. PLoS Comput. Biol. 2010, 6, 1-11. 